\title{
El Servicio Comunitario, Una Mirada Teórica
}

\author{
Autores: Carol del Carmen Terán González \\ Universidad Nacional Experimental "Rafael María Baralt”, UNERMB \\ carolterang@gmail.com \\ Trujillo, Venezuela \\ Wilmer José Araujo \\ Universidad Nacional Experimental "Rafael María Baralt”, UNERMB \\ wilmarprof@hotmail.com \\ Trujillo, Venezuela
}

\section{Resumen}

La presente investigación tuvo como objetivo fundamental, generar un constructo teórico bajo el enfoque ontoepistémico en la praxis del servicio comunitario para los estudiantes. El estudio se apoyó en las teorías de: Aprendizaje Servicio. Servicio Comunitario. La misma adoptó una mirada dentro del paradigma cualitativo en la esencia del fenómeno para describirlo, comprenderlo e interpretarlo en toda su extensión partiendo de un enfoque fenomenológico. Una vez entrevistados los cinco informantes claves se obtuvieron como resultado los aportes a través de juicios enunciativos desde su discurso, los mismos generaron seis picos teóricos que contribuyeron a enriquecer el estudio. Por último, se realizaron las reflexiones finales que dan respuesta a los propósitos del estudio.

Palabras clave: ontológico; epistémico; servicio comunitario. 


\title{
The Community Service, A Theoretical Look
}

\begin{abstract}
The present investigation had as fundamental aim generate a theoretical construct under the approach ontoepistémico in the practice of the community service for the students the study rested on the theories of: Learning Service. Community service. The same one adopted a look inside the qualitative paradigm in the essence of the phenomenon to describe it, to understand it and to interpret it in all his extension departing from an approach fenomenológico. Once interviewed five key informants obtained like proved the contributions across judgments enunciations from his speech, the same ones generated six theoretical beaks that helped to enrich the study. Finally there were realized the final reflections that give response to the intentions of the study.
\end{abstract}

Keywords: ontological; epistemic; community service.

Date Received: 22-08-2016 


\section{A manera de Introducción}

En este primer apartado de la investigación se hará el acercamiento a la problemática del fenómeno de estudio, el mismo contara en su recorrido discursivo las interrogantes, los propósitos, el contexto de estudio y la relevancia que tuvo la tesis como generación de conocimiento científico en la revolución de saberes hoy.

El afianzamiento de la cultura democrática es uno de los argumentos en las sociedades modernas, fundamentalmente por la creación de diferentes espacios para lo público; además, por las interrogantes sobre la complejidad que hoy se posesiona la gestión colectiva de los ciudadanos enunciada a través de las instituciones particulares. De allí que, la universidad es uno de los espacios oportunos para reconocer estas manifestaciones, discernir las representaciones para contribuir a estimular los valores sociales, asimismo como la organización de los ciudadanos. Para la UNESCO, (2005:26), en esta sociedad del conocimiento, las instituciones universitarias "se ven enfrentadas a un cambio radical sin precedentes en los esquemas clásicos de producción, difusión y aplicación de los conocimientos".

Por tal razón, la preservación de las sociedades modernas está determinada directamente por el grado de desarrollo a lograr y resulta lógico anticipar que en tanto más elevado y perfeccionado sea el desarrollo, más estables serán las sociedades. En tal sentido, es idóneo detectar y/o perfilar bajo qué parámetros de desarrollo se pretende impulsar la sociedad donde se habita, en concordancia con lo que sus integrantes aspiren colectivamente. Dicho de otra manera, se trata de compaginar el ideal societario con los modelos de desarrollo que mejor se adapten a la preservación y a la sustentabilidad.

Dicha preservación supone la disponibilidad de todos los recursos necesarios para que sus miembros puedan subsistir, esto por supuesto considera el uso racional y mensurado de los componentes de la naturaleza 
imprescindibles para la existencia ahora y en el futuro. Fernández, (2013) nos recuerda que fue en 1987 cuando por vez primera un organismo multilateral usó el término Desarrollo Sustentable; así mismo la Comisión Mundial para el Medio Ambiente y el Desarrollo, presidido por la investigadora Gro Harlem Brundtland lo definió como un modo de desarrollo que responde a las necesidades actuales sin comprometer las posibilidades que las futuras generaciones satisfagan las suyas.

En este sentido, se destaca la alta significación que tienen las instituciones educativas en virtud de sus cualidades y potencialidades para apoyar y fomentar el desarrollo. Los organismos educativos sobresalen por su función modeladora de individuos que se integrarán y convivirán en las sociedades; de manera especial hay que mencionar las universidades, las cuales poseen los recursos técnicos y gnoseológicos para mejorar las condiciones de vida de la colectividad. Resulta clara entonces la importancia que las entidades de educación universitaria propicien su inmersión activamente en los planes de desarrollo, pero esta participación debe ser orgánica, natural, así como voluntaria para lograr una respuesta favorable de las comunidades.

Evidentemente la formación de profesionales, la investigación y la extensión son funciones con una vinculación directa con los procesos de desarrollo de cada país que inciden directamente en el grado de prosperidad de cada región y comunidad donde se desplieguen; esto lo establece la Constitución de la República Bolivariana de Venezuela (CRBV):

Las obligaciones que correspondan al Estado, conforme a esta Constitución y a la ley, en cumplimiento de los fines del bienestar social general, no excluyen las que, en virtud de la solidaridad y responsabilidad social y asistencia humanitaria, correspondan a los o a las particulares según su capacidad. La ley proveerá lo conducente para imponer el cumplimiento de estas obligaciones en los casos en que fuere necesario. Quienes aspiren al ejercicio de cualquier profesión, tienen el 
deber de prestar servicio a la comunidad durante el tiempo, lugar y condiciones que determine la ley (Constitución de la República Bolivariana de Venezuela, Artículo 135).

Por ello, Gallardo (2013) destacaba el papel de las instituciones de educación universitaria al señalar que: "la universidad es una institución que pertenece a la sociedad, la cual ha estado vinculada a un tiempo y a un lugar cuyos saberes y estilos se adaptan a los retos y circunstancias de cada época". Así también la Ley Orgánica de Educación (LOE) establece entre los principios de responsabilidad social y la solidaridad el desarrollo de servicio comunitario expresado:

La responsabilidad social y la solidaridad constituyen principios básicos de la formación ciudadana de los y las estudiantes en todos los niveles y modalidades del Sistema Educativo. Todo y toda estudiante cursante en instituciones y centros educativos oficiales o privados de los niveles de educación media general y media técnica del subsistema de educación básica, así como del subsistema de educación universitaria y de las diferentes modalidades educativas del Sistema Educativo, una vez culminado el programa de estudio y de acuerdo con sus competencias, debe contribuir con el desarrollo integral de la Nación, mediante la práctica de actividades comunitarias, en concordancia con los principios de responsabilidad social y solidaridad, establecidos en la ley, (Ley Orgánica de Educación, 2009, art, 13).

Es pertinente resaltar que el Estado venezolano haya promulgado e implementado la Ley de Servicio Comunitario del estudiante de Educación Superior (LSCEES) (2005), la cual contempla que todos los estudiantes lleven a cabo un acercamiento con fines instruccionales, socio-afectivos en las comunidades, diseñando y/o participando en proyectos para el beneficio directo de las familias y miembros de la colectividad.

Este es un enfoque andragógico, basado en una experiencia de servicio solidario, en la cual los estudiantes, docentes y miembros de la comunidad 
aprenden, se forman y trabajan juntos para satisfacer necesidades comunitarias, potenciándose mutuamente los objetivos curriculares de un curso con los objetivos del servicio; considerando nuevos roles y relaciones para los tres actores directamente involucrados: el estudiante, el docente y lo socio-comunitario, tal como lo plantea Zuleta, citado por Quintero, (2008).

Como se puede observar, el servicio comunitario encierra mucho potencial para el fomento del desarrollo regional. A su vez, representa una enriquecedora oportunidad para lograr la satisfacción de las necesidades humanas de las familias y habitantes de las comunidades, al servir como instancia articuladora de esfuerzos apelando a la alta estimación que se tiene por las universidades en Venezuela.

La atención de la Ley del Servicio Comunitario en la educación universitaria es una realidad de espacios complejos en invariable construcción y reconstrucción que pasa por la resignificación de la red categorial: intervención comunitaria, comunidad, vínculos intersubjetivos, aprendizaje servicio, que sostienen todo un proceso de acción socio-comunitaria para desarrollar una cultura social de compromiso ético entre todos los que aquí participan.

Por ello, la implicación de ocupar conciencia solidaria y participativa; según Montero (2004:229), su concepción de participación comunitaria, concebida como "un proceso organizado, colectivo, libre, incluyente, en el cual hay una variedad de actores, de actividades y de grados de compromiso, que está orientada por valores y objetivos compartidos, en cuya consecución se producen transformaciones comunitarias e individuales".

La participación comunitaria, en cuanto a dimensión funcional e institucional, es el auténtico eje céntrico de la acción social que se hace necesario hoy para atender, de manera cohesionada, la gran deuda social de las instituciones de educación superior con su contexto. Este enfoque puede responder a un proceso solidario en actividades de concienciación, 
organización y formación comunitaria que conlleve no solamente a realzar la calidad de la educación, sino, paralelamente, a superar los niveles productores de la comunidad. En cuanto a la colaboración de las Instituciones de Educación Superior con la comunidad. De allí surgen las siguientes interrogantes.

¿Qué reflexiones surgen en relación a los hallazgos sobre la orientación bajo el enfoque ontoepistémico de los proyectos socio comunitario desde la perspectiva de los informantes clave para enunciar una aproximación teórica? Por lo cual se considera los siguientes propósitos:

\section{Propósitos de investigación. Propósito general.}

Generar un constructo teórico bajo el enfoque ontoepistémico en la praxis del servicio comunitario para los estudiantes de la Universidad de los Andes, Núcleo "Rafael Rangel”, estado Trujillo.

\section{Propósitos específicos.}

Definir las disciplinas que operan en el plano ontológico en la aplicación de proyectos socio-comunitarios bajo la Ley de Servicio Comunitario del Estudiante de Educación Universitaria.

Enunciar una aproximación teórica que permita la orientación bajo el enfoque ontoepistémico los proyectos socio-comunitarios en la praxis del Servicio Comunitario del Estudiante de Educación Superior.

\section{Referentes Teóricos}

Este enunciado es parte fundamental del presente apartado ya que en al mismo se hará un recorrido por los aspectos teóricos relevantes para el estudio, en un primer instante se revisaron los aportes de las teorías del aprendizaje en la educación, luego se planteó todos los discursos 
conceptuales que sustentan lo ontoepistémico en el servicio comunitario dentro de su práctica, estableciendo así los supuestos teóricos con sus precursores principales.

\subsection{Visiones teóricas del aprendizaje en la educación.}

Se comenzará con el constructivismo, el mismo sostiene que las personas activamente construyen conocimiento mientras interactúan con su ambiente. Esto es postulado por el psicólogo suizo Piaget, (1970:92), quien expresa que el aprendizaje como en la generación de estructuras cognoscitivas que se crean a través de la modificación de los reflejos iníciales del recién nacido y que se va enriqueciendo a través de la interacción del individuo con el medio. Estas estructuras se encargan de adquirir la nueva información a través de dos procesos básicos: la asimilación y la acomodación.

En este sentido, surge una perspectiva teórica que complementa el enfoque de Piaget, desarrollada por el psicólogo ruso Vygotsky (ob.cit.), y que enfatiza en la interacción social como factor clave para el aprendizaje y la transmisión de la cultura. En esto, la ley genética general del desarrollo cultural, como la denominó Vygotsky (ob.cit.), ofrece una explicación de la relación entre el aprendizaje y la interacción social, elemento fundamental en la práctica del servicio comunitario donde los elementos socioculturales están inmersos en todos sus actores.

En este sentido los llamados conocimientos previos y la experiencia de quien aprende, resultan determinantes en la construcción de nuevos conocimientos, donde la triada Tutor, prestador de servicio y comunidad adquiere saberes desde la internalización propia de todos los actantes, con una aprehensión del conocimiento de forma holística e integral, producto de la cotidianidad que se vive durante la puesta en práctica del servicio comunitario. 


\subsection{Servicio Comunitario.}

El servicio comunitario es una idea que existía desde la época de Republica Romana, donde sus ciudadanos estaban unidos por derechos legales y cargas imperativas. En los Estados Unidos los primeros indicios surgen en 1906, cuando Williams James planteaba la necesidad un servicio civil moral y en 1966 John F. Kennedy planteó a los ciudadanos de su nación el hecho de no preguntarse que esperaban de su país, sino que podían aportar ellos en su beneficio. En México, se lleva a cabo el servicio desde 1910, y se presta nivel universitario con una duración de 400 horas. En costa Rica, desde 1975, y se presta por 150 horas. En Brasil, no existe el Servicio comunitario como tal, pero hay un programa similar con duración de 6 meses.

En Colombia se incorpora como parte del pensum universitario en el año 1991 y tiene una duración de un semestre académico. Un interesante programa se formuló en Inglaterra, ideado por David Blunkett entre 19972001 , donde se plantea que los estudiantes entre 5 y 18 años, ocupen un $5 \%$ de su tiempo escolar en actividades orientadas al aprendizaje y la práctica de valores ciudadanos, así como también sobre reciclaje y conservación del medio ambiente.

En Uruguay se aplica desde 2002 el programa aprendiendo juntos, promovido por voluntarios y que involucra a estudiantes. En Venezuela, oficialmente se inicia la práctica del servicio comunitario, como actividad universitaria, desde el año 2005 donde se señalan las actividades que deben desarrollarse en las comunidades por los estudiantes de Educación Superior que cursan estudios de formación profesional, para cooperar con su participación al cumplimiento de los fines del bienestar social.

Se observa así una metodología viable a ser aplicada que orienta al participante a interactuar con los grupos humanos localizados en las comunidades, donde el acatamiento de la disposición en el cumplimiento de dicha Ley está basado en estrategias de aprendizaje en servicio 
implementadas con proyectos que les brindan oportunidad de formación y crecimiento organizativo. Al mismo tiempo mejora la formación de los estudiantes para que se conviertan en profesionales con visión inclusiva del país y con un compromiso social solidario, efectivo y duradero, mediante la inserción curricular de un eje socio-transversal en cada una de las carreras ofertadas, a las cuales se les agrega como requisito su cumplimiento para egresar con el título correspondiente.

Partiendo del preámbulo anterior es preciso señalar que la Ley del Servicio Comunitario del Estudiante de Educación Superior. Venezuela, 2005. En el artículo 4to de esta ley se encuentra la definición oficial del Servicio Comunitario:

A los efectos de esta Ley, se entiende por Servicio Comunitario, la actividad que deben desarrollar en las comunidades los estudiantes de educación superior que cursen estudios de formación profesional, aplicando los conocimientos científicos, técnicos, culturales, deportivos y humanísticos adquiridos durante su formación académica, en beneficio de la comunidad, para cooperar con su participación al cumplimiento de los fines del bienestar social, de acuerdo con lo establecido en la Constitución de la República Bolivariana de Venezuela y en esta Ley.

En un período que no podrá ser inferior a 120 horas, los actores participantes (estudiantes/prestadores del servicio, los docentes/asesores y las instituciones de educación superior) desarrollan de manera organizada y sistémica todas las actividades que se deriven del servicio comunitario. En los fines, se resalta el fomento de la solidaridad y el compromiso con la comunidad; integrar las instituciones de Educación Universitaria a las comunidades; y fortalecer la experiencia de los estudiantes a través del aprendizaje servicio. 


\subsection{Aprendizaje Servicio.}

Tal como se explicó con anterioridad, el Aprendizaje Servicio es un enfoque metodológico con fundamentos axiológicos, pues concibe al prestador como un sujeto que se puede integrar al entorno durante el ejercicio educativo del que toma parte, articulando y orientando el proceso de transformación de la realidad de las comunidades. Al mismo tiempo, tal proceso se puede caracterizar como dialéctico en virtud de la confrontación de los conceptos y teorías que sustentan su formación académica con los elementos objetivos y subjetivos típicos de la realidad, y, en otra medida, la cualidad dialéctica se expresa en tanto se va dando la transformación del sujeto o sujetos participantes, así como también de los espacios en donde el enfoque se concretiza.

Rifkin analizó el impacto del Aprendizaje Servicio en los retos del nuevo milenio, y lo ha definido como:

Un antídoto esencial para el mundo crecientemente aislado de la realidad virtual y simulada que los niños experimentan en clase y en sus hogares, frente al televisor o la computadora. Darles a los jóvenes una oportunidad para una participación más profunda en la comunidad los ayuda a desarrollar el sentido de la responsabilidad, solvencia personal, alienta la autoestima y el liderazgo, sobre todo, permite que crezcan y florezcan el sentido de creatividad, iniciativa y empatía, (Rifkin, 1996).

\subsection{Principios del Aprendizaje Servicio.}

Siendo el aprendizaje en servicio un enfoque metodológico, busca transformar positivamente una realidad social a partir de un acto simultáneo de reconocimiento multilateral de actores participantes, toda la experiencia tendrá como fundamentos los siguientes principios: alteridad, solidaridad, responsabilidad social, igualdad, cooperación, corresponsabilidad, 
participación ciudadana y asistencia humanitaria; los cuales se describen seguidamente.

En primer lugar, se tiene el principio de alteridad, el cual etimológicamente viene del latín "Alter" que significa el "Otro". Implica el reconocimiento del otro como un sujeto que comparte un espacio significativo con vínculos en el lenguaje, la cultura y las creencias. Sociológicamente es una categoría importante ya que facilita la coexistencia de individuos y precondiciona el sentido colectivo, pues sólo pueden asociarse personas que se aceptan como tales. En el marco del aprendizaje servicio supone forzosamente la introspección individual, el entendimiento del sí mismo como ser individual y la toma de conciencia de los otros que nos rodean. Prevé la concesión incondicional a las características del otro; el reconocimiento digno y absoluto de la realidad ajena y la práctica de la aceptación.

Con respecto a la solidaridad, surge como valor y premisa fundamental de funcionamiento como elemento para direccionar las voluntades de los individuos que la conforman bajo la condición de estimar al sujeto como integrante de un colectivo, del que participa, representa y afecta.

En cuanto a la responsabilidad social, surge de la construcción fáctica, lo que implica la constatación del hecho. A los efectos del servicio comunitario, la responsabilidad social apertura a los institutos de educación superior a lo interno de las comunidades y la apropiación de espacios que permitan alcanzar proyectos de desarrollo integradores con base en principios constitucionales como el bien común y la convivencia.

En su ejercicio, la responsabilidad social universitaria deberá proveer los mecanismos para la utilización de estrategias propias del aprendizaje servicio en la actividad comunitaria, aupando así una conducta ética adecuada a la responsabilidad asumida por y con todos los actores involucrados. Surge entonces con la obligatoriedad del servicio, la mejor oportunidad para formar en valores para lo recíproco, en democracia y tolerancia a la diversidad. 
También se encuentra el principio de igualdad; el cual se define como la ausencia total de discriminación entre los seres humanos, en lo que respecta a sus derechos. Para el servicio comunitario, representa el factor orientador de la conducta de los estudiantes/prestadores del servicio, ya que todo el potencial de beneficios para los miembros de la comunidad no puede estar bajo ningún concepto condicionados, y menos aún, ser objeto de discriminación que lesionen la dignidad de las personas y vulneren sus derechos fundamentales.

\section{Sendero metodológico.}

La presente investigación tiene como propósito generar un constructo teórico bajo el enfoque ontoepistémico en la praxis del servicio comunitario a partir de los significados de las experiencias de estudiantes prestadores de servicio y su docente asesor sobre los juicios valorativos en la cotidianidad de su praxis.

Partiendo de esta intencionalidad investigativa, surge la inquietud de indagar a profundidad sobre la perspectiva que los estudiantes prestadores de servicio comunitario poseen en cuanto a los juicios valorativos que emiten durante su praxis de servicio. Sobre la base de esta reflexión metodológica, se considera la ubicación del presente estudio dentro del paradigma cualitativo, por cuanto permite profundizar en la esencia del fenómeno para describirlo, comprenderlo e interpretarlo en toda su extensión. Describe el hecho en el que se desarrolla el acontecimiento, la investigación cualitativa trata de estudiar a profundidad un fenómeno, por ello acude a la descripción contextual de los hechos, es decir que la indagación la efectúa el investigador en el propio teatro de operaciones. 
Figura 1. Soporte metodológico

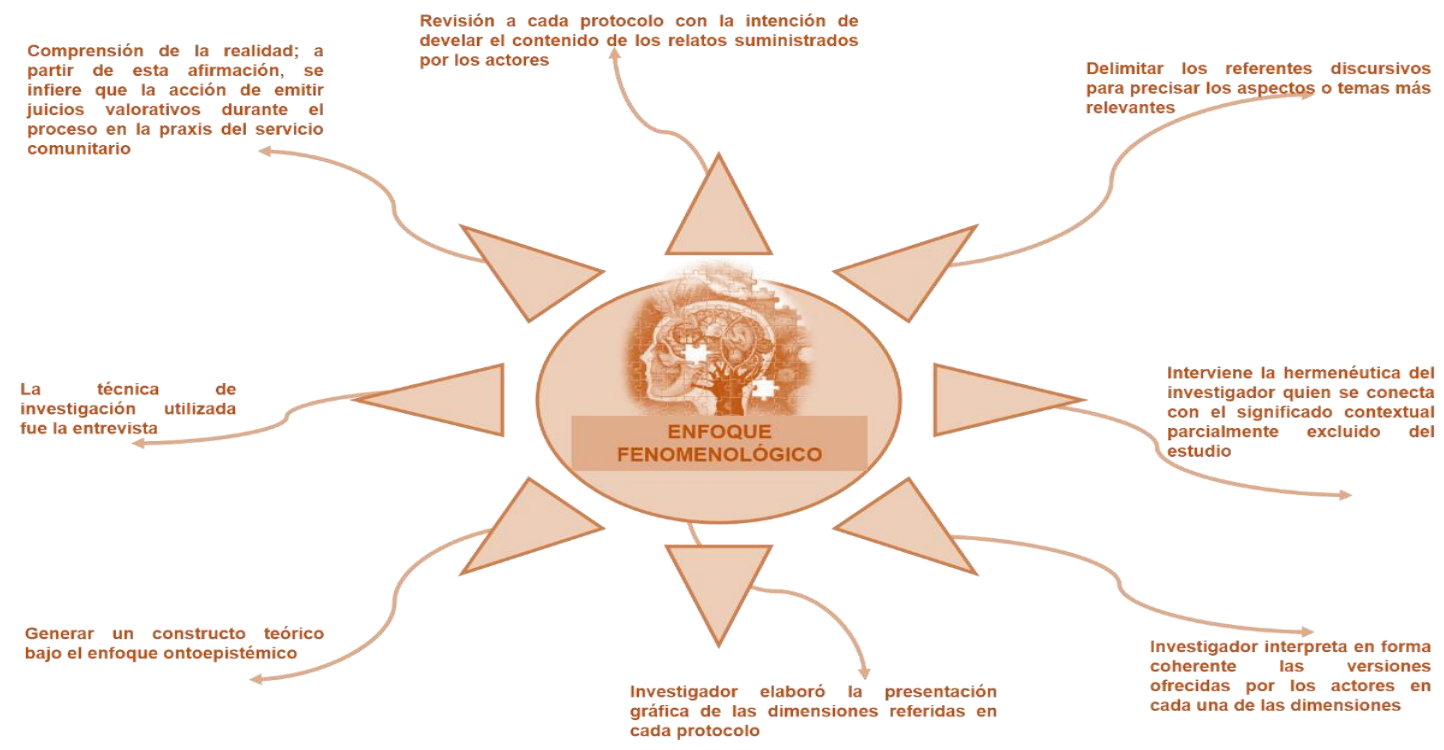

Fuente: Terán y Araujo (2016)

Para los efectos de este estudio, la descripción de significados de los estudiantes en servicio y su asesor, los informantes les atribuyen a los juicios valorativos en la praxis del servicio comunitario, se hace en función de la reflexividad de los propios actores.

Se planteará el análisis a partir de una triangulación de la información que contó con los juicios emitidos por los informantes, las dimensiones y los atributos para luego generar la aproximación teórica pertinente de acuerdo a la realidad ontoepistémico en la praxis del servicio comunitario.

\section{Construcciones teóricas ontoepistémicas del servicio comunitario en la praxis social.}

A partir de los enunciados de los informantes, la ojeada del otro y la fundamentación teórica revisadas permiten el ejercicio de la construcción de este estadio, el mismo constituye una aproximación teórica desde una mirada interpretativa al fenómeno estudiado, que consistió en el acercamiento a los 
elementos ontoepistémico que coexisten en la praxis del servicio comunitario, de allí que se vislumbren seis representaciones discursivas como propuesta en cuanto al estudio.

Para Martínez (2015:154) la teoría es por tanto, un modelo ideal, que ofrece una estructura conceptual, inteligible, sistémica y coherente para ordenar fenómenos. Así que este apartado mostrara una visión enunciativa, que se vincula directamente con el fenómeno investigado, no es una verdad absoluta, pero sin embargo es una nueva propuesta desde el Sujeto cognoscente que la propone.

Figura 2. Construcciones teóricas

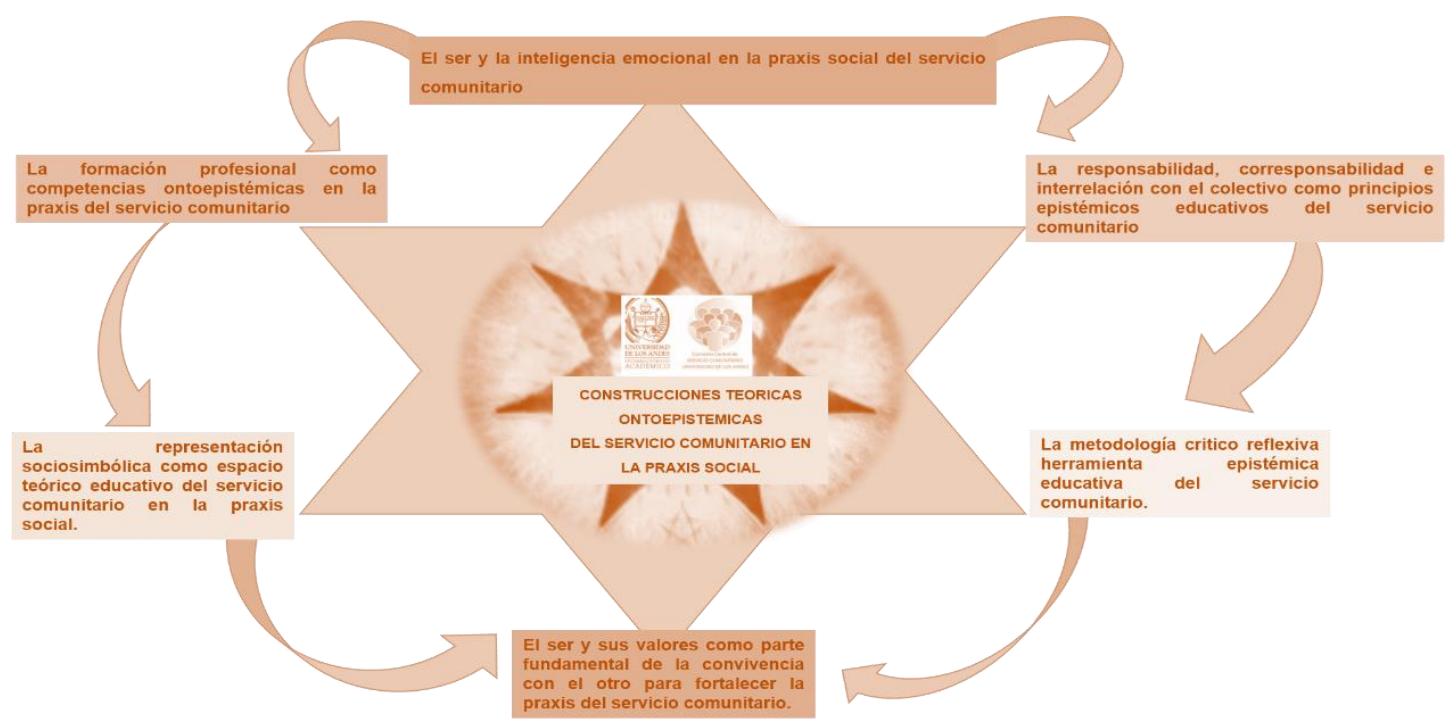

Fuente: Araujo, Terán y Furguerle (2016)

\section{Reflexiones finales}

Partiendo de la experiencia durante la investigación un Acercamiento Ontoepistémico a la praxis del servicio comunitario y como fruto de la labor durante este estudio, considero oportuno realizar una serie de reflexiones que sobre el proceso y producto del tema se generaron. 
Lo anterior se logra, teniendo en cuenta que lo ontológico es aquello que tiene inmerso al Ser, dentro de las normas que lleva inmerso el servicio comunitario en el desarrollo de los proyectos, los informantes plantearon la comunicación asertiva, la inteligencia emocional y los principios axiológicos internos de cada actor social. "quienes prestan servicio comunitario como quienes los orientan deben tener empatía con los otros, saber utilizar herramientas de la comunicación como el asertividad y la inteligencia emocional para poderse involucrar con el otro" (informante 02) Esto implica que entre las disciplinas que arropan el servicio comunitario debe fortalecerse el lenguaje y la comunicación como base para la relación con el otro.

También el hecho de la asertividad y los valores como ejes de conocimiento de gran valoración para la convivencia y el quehacer comunitario, de forma que se fortalezcan los vínculos e interacción con el otro

Por otro lado, se tenía como segundo propósito, determinar las estrategias usadas por los docentes desde el enfoque epistemológico en la aplicación de proyectos socio comunitario bajo la Ley de Servicio Comunitario del estudiante de Educación Universitaria, para ello las apreciaciones obtenidas en cuanto a las estrategias epistémicas hubo total coincidencia en la aplicación de una metodología critico reflexiva que lleve a la transformación de la realidad y las problemáticas a solventar.

Es de hacer notar que el prestador de servicio debe hacer uso de herramientas epistémicas como la teoría critica reflexiva desde la investigación acción transformadora para que se dé una verdadera apropiación de conocimientos y saberes desde todos los actantes sociales, mediante la construcción, apropiación y transformación de la realidad.

Por otro lado, el propósito final de la investigación era enunciar una aproximación teórica que permita la orientación bajo el enfoque ontoepistémico los proyectos socio-comunitarios en la praxis del servicio comunitario del estudiante de Educación Superior. En cuanto a esta premisa 
es de hacer notar que partiendo de los juicios valorativos y hallazgos se realizó una estrella de seis picos, cada uno contentivo de una aproximación teórica generada de la construcción de todos los actores, tanto los sujetos cognoscibles como el sujeto cognoscente.

Como puede apreciarse el constructo investigativo genero seis posturas que sustentan lo ontoepistémico en la praxis del servicio comunitario, tres elocuciones incorporan los aspectos del ser y los otros tres los elementos desde la producción de saberes.

Es preciso señalar que los valores y elementos axiológicos son fuente de vitalidad en el servicio comunitario, además de considerar lo axiológico plantea las relaciones sociosimbolicas dentro del espacio comunitario, entes que nutren la riqueza y diversidad del otro que convive en un espacio determinado bajo una realidad concreta y que será el escenario donde el prestador de servicio abordará su quehacer comunitario.

Por último, es preciso señalar que el trabajo es un abreboca de muchas concepciones, teorías, discursos y espacios abordados por los actores sociales, quienes alimentaron el producto investigativo aquí presentado y que además deja una puerta abierta a nuevas producciones y construcciones de saberes desde la vivencia y relación teórico práctica del ser con el otro.

\section{Referencias}

Constitución de la República Bolivariana de Venezuela (1999). Gaceta Oficial Extraordinaria $\mathbf{N}^{\circ} 36.860$ de fecha 30 de diciembre. Caracas, Venezuela: Asamblea Nacional Constituyente.

Cecchi, N. (2006). La Experiencia Latinoamericana. Aprendizaje Servicio en Educación Superior. Seminario Internacional "Responsabilidad Social Universitaria. Caracas. 
Díaz, Z. (2004). La Significación Social de la Transformación Universitaria. En La Universidad se Reforma IV. Editorial UCV, ORUS, UNESCO, UPEL, MES. Caracas, pág. 160.

Francia, Aranaga, y Fernández (2009). La Construcción del conocimiento científico. Filosofía y Ética de la Ciencia. España: Narcea.

Gimeno, S. (2001). Educar y convivir en la cultura global. Madrid: Editorial Morata.

Guba, E. (2007). El Diálogo del Paradigma Alternativo. Newbury Park: Sage.

Guijarro, M., y Chávez, J. (2006). Gerencia Universitaria. Documento presentado en la Universidad del Zulia. En Revista Venezolana de Gerencia (RVG), Año 11. № 34, 2006, 201-220.

Herrera, C. (2005). Consideraciones sobre la Ley de Servicio Comunitario del Estudiante de Educación Superior. Ponencia presentada en la Jornadas de Derecho Procesal y Administrativo, Universidad Católica Andrés Bello.

Hurtado, J. (2008). Metodología de la Investigación. Una comprensión Holística. Caracas, Venezuela: Ediciones Quirón.

Kliksberg, B. (2004). Capital Social, una idea poderosa ¿Cómo enseñarla? Capital Social, Ética y Desarrollo. Caracas: OPSU, UNIMET, MES.

Ley de Servicio Comunitario del Estudiante de Educación Superior (2005).

Gaceta Oficial № 39.272, 14 de septiembre. Venezuela.

Ley de Universidades (1970). Gaceta Oficial de la República de Venezuela.

Extraordinaria No.1429, del 8 de septiembre. Caracas, Venezuela.

Ley Orgánica de Educación (2009). Gaceta Oficial de la República Bolivariana de Venezuela. Caracas, Venezuela.

Martínez, M. (2015). Cómo hacer un Buen Proyecto de Tesis con metodología Cualitativa. Trillas. México. 
Méndez, L. (2009). La Escuela Técnica como Centro Comunitario para la Paz y la Productividad: Una experiencia de cambio organizacional escolar. Ponencias presentada en el marco de la Jornada Rol de la Educación Superior, en la Universidad Central de Venezuela.

Montero, M. (2004). Introducción a la Psicología Comunitaria. Desarrollo, Conceptos y Procesos. Editorial Paidós. SAICF, Argentina, pág. 229.

Molina, J. (2008). Presentación. El análisis de redes sociales en España y Latinoamérica. Revista Redes, vol. I, 2001. Revista Redes. España.

Popkewitz, T. (2005). Paradigma e Ideología en Investigación Educativa. Mondadori. España.

Tapia, C. (2002). Aprendizaje-Servicio en Educación Superior. La Experiencia Comunitaria. Madrid, España: Editorial Muralla.

Toledo (2009). Pensamiento complejo y educación. Madrid: Ediciones de La Torre.

UNESCO (1998). Declaración sobre la Educación Superior en el Siglo XXI. Conferencia Mundial sobre la Educación Superior en América y El Caribe. Paris: Edición UNESCO. 


\section{Carol del Carmen Terán González}

e-mail: carolterang@gmail.com

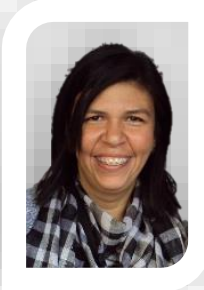

Nacida en la ciudad de Valera Edo Trujillo. Curso estudios de Doctorado en Educación en la Universidad Rafael María Baralt, posee Maestría en Docencia para la Educación Superior en la misma universidad, Maestría en Literatura Latinoamericana en la Universidad de los Andes y Licenciatura en Educación Mención Castellano y

Literatura ULA-NURR investigadora del Centro de investigaciones literarias y lingüísticas Mario Briceño Iragorry. (CILL) ULA-NURR, Jefe de la unidad de investigación y proyecto de la Casa de Historia Trujillo, articulista e investigadora con 14 años de servicio en el área de educación y literatura. Ponente en eventos nacionales e internacionales. Docente invitada en pregrado y Postgrado, ULA, UNERMB, UNESR. Coordinadora de la Línea de investigación Memoria, Educación y Discursos emergentes (UNERMB), miembro de la Línea de investigaciones Ciudadanía, hermenéutica y proyectos sociales. (UNERS). Ha realizado artículos en distintas revistas arbitradas del país entre ella Sapiencia, Cifra Nueva, Revista de Cultura Centro Nacional de Historia, Revista de Ingeniería UVM, entre otras. 


\section{Wilmer José Araujo}

e-mail: wilmerprof@hotmail.com

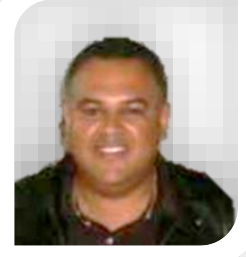

Nacido en la ciudad de Valera Edo Trujillo. Doctorante en Educación Universidad Rafael María Baralt, Maestría en Docencia para la Educación Superior en la misma universidad, Licenciado en Educación Mención Química ULA-NURR. Docente de Media General del Ministerio de Educación con 17 años de

servicio. Docente invitado postgrado UNERMB. Desempeño funciones como docente de pregrado en la Universidad Alonso de Ojeda. Miembro de la Línea de investigación Memoria, Educación y Discursos emergentes (UNERMB).

El contenido de este manuscrito se difunde bajo una Licencia de Creative Commons ReconocimientoNoComercial-Compartirlgual 4.0 Internacional 